\title{
Models of Geometrically "Stabilized" Laser Cavity
}

\author{
A. Aissani ${ }^{1, a}$, S. Leghmizi ${ }^{1}$ and K. Battou ${ }^{2}$ \\ ${ }^{1}$ Laboratory of Quantum Electronics, Faculty of Physics-USTHB, B.P.32,El Alia, 16111, \\ Bab Ezzouar, Algiers, Algeria. \\ ${ }^{2}$ CSViamonde, 116 Cornelius Parkway, Toronto M6L 2K5, Canada
}

\begin{abstract}
The Lamb-dip characterizing the line shape emitted by a gas laser is often used in spectroscopy of materials as a reference for frequency measurements. For such lasers, the frequency control is performed on the Lamb-dip. It is therefore essential, for accurate measurements, that its frequency matches with the laser resonance frequency. This is only possible if the emitted line shape is symmetrical, which is not usually the case. Indeed, the lens effects induced in the laser amplifying medium, which are due to the population and the saturation inhomogeneities, generally produce an asymmetrical emitted line shape. So, the frequency of the Lamb-dip is shifted compared to the central frequency.

In this work, we will first revisit the model given in the literature, in order to highlight the limit of its validity, and then we will propose through an appropriate choice of the cavity geometry, a "stabilized" cavity model giving rise to a symmetrical line shape even when the control parameters vary.
\end{abstract}

\section{Introduction}

In several areas of physics [1-4], the Lamb dip characterizing the line shape of a gas laser is used as a reference for frequency and length measurements. These measurements would be more accurate if the line shape was symmetrical, in which case the center frequency of the laser would coincide with that of the Lamb dip [5]. In fact, several causes are at the origin of this asymmetry [6-9]. These combine to create population and saturation inhomogeneities, which induce lens effects that modify the wave mode diameter. The variations as a function of the frequency of these parameters are then at the origin of the asymmetry of the line [10]. However, geometrical parameters [1, 11] can modulate the competition between these two types of antagonistic heterogeneities and favor one or the other. It is for this purpose that several models of cavities, called "stabilized", have been proposed [12-13].

In this work, we will first revisit one of these two models [12] and then, based on the perturbed Gaussian beam theory [14] and by making a judicious choice of geometric parameters, we will propose a geometric cavity structure giving a symmetrical output line shape, even when control parameters vary.

\section{Theoretical Model}

The theoretical calculation described below concerns the laser cavity shown schematically in Fig.1. It consists of a plane mirror $\mathrm{M}_{1}$ and a curved mirror $\mathrm{M}_{2}$ of curvature radius $\mathrm{R}$, separated by a distance $\mathrm{D}$

\footnotetext{
${ }^{\text {a }}$ Corresponding author : ah.aissani@yahoo.com
} 
and placed respectively at $z_{1}$ and $z_{2}$, an amplifier tube of length $L_{g}$, placed between $z_{3}$ and $z_{4}$, and a diaphragm of diameter $2 b$ placed on the side of the output mirror $\mathbf{M}_{2}$. The mirrors reflective powers are $r_{A}$ and $r_{B}$, respectively. In order to have a fixed linear polarization, the ampifier tube is closed by two Brewster angle windows.

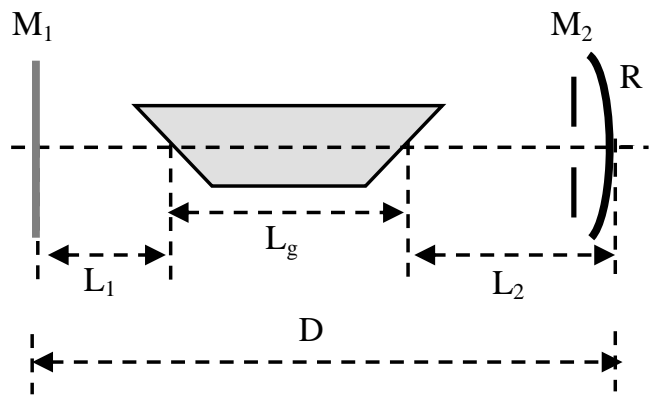

Figure 1: Schematic representation of the cavity

The amplitude of the resonant field is obtained from the propagation equation. Its expression, for the forward wave, can be written in the form:

$$
E(\rho, \zeta)=\frac{E_{0}}{1+2 i \zeta} \exp \left[i\left(k \frac{\omega}{c} W_{0}^{2}\right) \zeta\right] \exp \left[\frac{-\rho^{2}}{1+2 i \zeta}\right] \exp \left[-i\left(p-\frac{\rho^{2}}{2} q\right)\right]
$$

Where: $E_{0}$ is a constant, $k$ the propagation constant, $\mathrm{p}$ and $q$ represent, respectively, the perturbations of the longitudinal and transversal parameters of the wave, $2 W_{0}$ is the mode diameter at the beam waist, and $(\rho, \zeta)$ are the reduced coordinates connected to the cylindrical coordinates $(r, z)$ by:

$$
\rho=\frac{r}{W_{0}} ; \zeta=\frac{c z}{\omega W_{0}^{2}}
$$

The proposed cavity is composed of three regions. In region 1: $z_{1} \leq z \leq z_{3}$, in region 2: $z_{3} \leq z \leq$ $z_{4}$ and in region $3, z_{4} \leq z \leq z_{2}$. The propagation constant $k$, it is equal to $\left(\frac{\omega}{c}\right)$ in the empty regions 1 and 3 and $k_{g}$ in the region 2 containing the active medium. In addition, the oscillating field in the cavity is composed of a forward and a backward waves, respectively indexed " $f$ " and " $b$ " in the next equation. The equation (1) takes the explicit form below:

$$
{ }^{\mathrm{m}} E_{f, b}(\rho, \zeta)=\left(\frac{{ }^{\mathrm{m}} E_{0 f, b}}{1 \pm 2 i \zeta}\right) \exp \left[ \pm i\left(k \frac{\omega}{c} W_{0}^{2}\right) \zeta\right] \exp \left[\frac{-\rho^{2}}{1 \pm 2 i \zeta}\right] \exp \left[-i\left({ }^{\mathrm{m}} p_{f, b} \mp \frac{\rho^{2}}{2} \mathrm{~m}_{f, b}\right)\right]
$$

The upper sign in the symbols " \pm " or "Ғ" corresponds to the forward wave and the lower one to the backward wave.

This expression allows us to easily determine the field at the output of the cavity. We will assume that the transmission coefficients at the boundary of two neighboring regions are equal to 1 . In addition, the continuity relations of the field make it possible to write:

$$
E_{f, b}\left(0, \zeta_{j}\right)={ }^{\mathrm{m}} E_{f, b}\left(0, \zeta_{j}\right)
$$

Where $\zeta_{j}$ is the reduced coordinate at the border of two neighboring regions $(j=3$ or 4$)$ and $\mathrm{m}$ is the region number.

Given these considerations, we obtain at $\zeta_{2}$ :

$$
\begin{aligned}
& { }^{3} E_{f}\left(\rho, \zeta_{2}\right)=\left[\frac{1+2 i \zeta_{1}}{1+2 i \zeta_{2}}\right]{ }^{1} E_{f}\left(0, \zeta_{1}\right) \exp \left[i\left(\frac{\omega}{c} W_{0}\right)^{2}\left(l_{1}+l_{2}\right) \zeta+i \frac{\omega}{c} W_{0}^{2} k_{g} l_{g}\right] \exp \left(-i p_{F}\right) \\
& \cdot \exp \left[\frac{-\rho^{2}}{1+2 i \zeta}\right] \exp \left(-i \frac{{ }^{\prime} q_{f, b}}{2} \rho^{2}\right)
\end{aligned}
$$


Where $p_{F}=\left({ }^{1} p_{f_{3}}-{ }^{1} p_{f_{1}}\right)+\left({ }^{2} p_{f_{4}}-{ }^{2} p_{f_{3}}\right)+\left({ }^{3} p_{f_{2}}-{ }^{3} p_{f_{4}}\right)$ and $l_{1}, l_{2}, l_{g}$ are the reduced lengths corresponding respectively to $\mathrm{L}_{1}, \mathrm{~L}_{2}$ and $\mathrm{L}_{\mathrm{g}}$.

The corresponding intensity which is proportional to the square of the modulus of the electric field amplitude is defined as:

$$
I_{f}\left(\rho, \zeta_{2}\right)=\left[\frac{1+4 \zeta_{1}^{2}}{1+4 \zeta_{2}^{2}}\right] I_{f}\left(0, \zeta_{1}\right) \exp \left[-2\left(\frac{\omega}{c} W_{0}^{2}\right) k_{g}^{i} l_{g}+2 p_{F}^{i}\right] \exp \left[-\frac{2}{1+4 \zeta_{2}^{2}} \rho^{2}\right] \exp \left(-{ }^{3} q_{f_{2}}^{i} \rho^{2}\right)
$$

Where $k_{g}^{i}, p_{F}^{i}$, and $q_{f_{2}}^{i}$ designate the imaginary parts of these different quantities.

Since the asymmetry of the line shape depends on the position of the detector at the output of the laser [15], we will be interested in the emitted total power $P_{f}\left(\zeta_{2}\right)$. If $b$ is the radius of the diaphragm (the corresponding reduced length is then $\left(\frac{b}{W_{0}}\right)$, we have :

$$
P_{f}\left(\zeta_{2}\right)=W_{0}^{2} \int_{0}^{2 \pi} \quad d \varphi \int_{0}^{\frac{b}{W_{0}}} \quad I_{f}\left(\rho, \zeta_{2}\right) \rho d \rho
$$

After some analytical calculations, we obtain:

$$
\begin{aligned}
& P_{f}\left(\zeta_{2}\right)=\pi W_{0}^{2} I_{f}\left(0, \zeta_{1}\right)\left[\frac{\left(1+4 \zeta_{2}^{2}\right)}{2+\left(1+4 \zeta_{2}^{2}\right)^{3} q_{f_{2}}^{i}}\right] \exp \left[-2\left(\frac{\omega}{c} W_{0}^{2}\right) k_{g}^{i} l_{g}+2 p_{F}^{i}\right] \\
& {\left[1-\exp \left[-\frac{b^{2}}{W_{0}^{2}} \frac{2+\left(1+4 \zeta_{2}^{2}\right)^{3} q_{f_{2}}^{i}}{\left(1+4 \zeta_{2}^{2}\right)}\right]\right]}
\end{aligned}
$$

This equation shows that the total power $P_{f}\left(\zeta_{2}\right)$ at the side 2 of the laser depends upon the total power $\left(\pi W_{0}^{2} I_{f}\left(0, \zeta_{1}\right)\right)$ at the side 1 , modified by the transmission factor across the diaphragm (last term in square brackets), the gain by the exponential containing $k_{g}^{i}$ and the saturation term $2 p_{F}^{i}$, and the geometric parameters of the cavity.

The obtained expression contains the longitudinal and transverse perturbations of the Gaussian beam that can be obtained using their evolution equations and continuity conditions at the boundary of two regions. We will use the equation 8 , in our numerical calculations to plot the line shapes at the output of the laser.

\section{Numerical Results}

The numerical calculation concerns a single mode He-Ne laser operating at $3.39 \mu \mathrm{m}$ and it's based on the perturbed Gaussian beam model [14]. The cavity (see Fig.1) is hemispherical, of length D. The reflection coefficients are $r_{A}=1$ for the plane mirror and $r_{B}=0.8$ for the concave one. The curvature radius for the output mirror $\mathrm{M}_{2}$ is equal to $R=600 \mathrm{~mm}$. The amplifier tube, of length $L_{g}$, is closed by two windows at Brewster angle. In order to select the Gaussian fundamental mode $\mathrm{TEM}_{00}$, the diameter $2 b$ of the diaphragm was chosen to be equal to about 1.2 times the mode diameter at the laser output [12].

In a first step, we wanted to verify the model of stabilized cavity proposed by the authors of the reference [12]. In this model, the active medium is assumed to a thin lens centered in the cavity. So, the approach followed is to assume that the amplifier tube is centered in the cavity to find a value $G_{0}$ of the gain which gives a symmetrical line shape and, if so, to test other gain values, below and above $G_{0}$, to see if the symmetry of the line shape is preserved. The length $D$ of the cavity is fixed only by the value $R$ of the curvature radius of the curved mirror through the relation $D=\frac{4}{5} R$ [12], which corresponds in our case to $D=480 \mathrm{~mm}$. In the following the length of the active tube is fixed to $L_{g}=200 \mathrm{~mm}$.

The Fig. 2 shows, for three appropriately chosen gain values, the total power at the laser output as a function of the reduced frequency $x=\left(\omega-\omega_{0}\right) / \Gamma$, where $\omega_{0}$ is the laser center frequency and $\Gamma$ is 
the Doppler half-width. We see that the line shape is symmetric for $G_{0}=1.86$, as predicted by the authors. On the other hand, for a smaller gain $G=1.80$, the saturation inhomogeneity outweighs the population one. The asymmetry of line shape has a maximum on the low frequencies side (LF asymmetry type), while, for a higher gain $G=2.10$, population effects outweigh the saturation one and the maximum is on the high frequencies side (HF asymmetry type). This shows clearly the switch of the asymmetry from one type to the other when the gain varies. We conclude that for $G_{0}$, population and saturation effects compensate each other, giving a symmetrical line shape. But this symmetry disappears as soon as the gain $G$ changes. It is therefore obvious that the proposed model does not allow to obtain a so called "stabilized cavity".

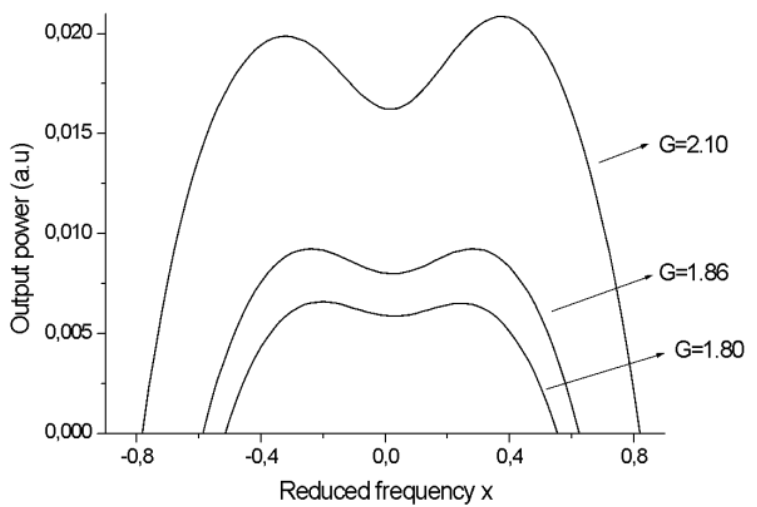

Figure 2: The line shape of the laser output versus the reduced frequency $x$

To achieve the main part of this paper, we will now focus on the study of the case where the gain varies, and we will clearly show that this variation does not affect the asymmetry, in contrary to the previous model. After several simulations, the cavity length is set to $D=500 \mathrm{~mm}$ and contains an active tube of length $L_{g}=150 \mathrm{~mm}$. The rest of the settings are unchanged. The gain $G$ has been set to 2.1. This choice is motivated by the fact that this value gives rise to a switch of the asymmetry when the position of the active tube varies and gives line shapes with a pronounced Lamb-dip.

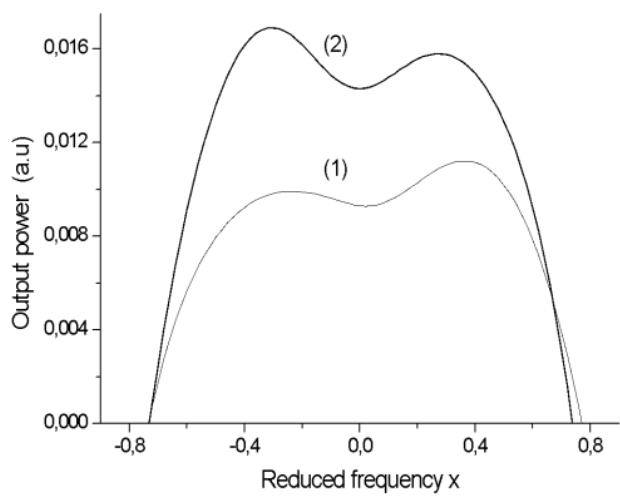

(a)

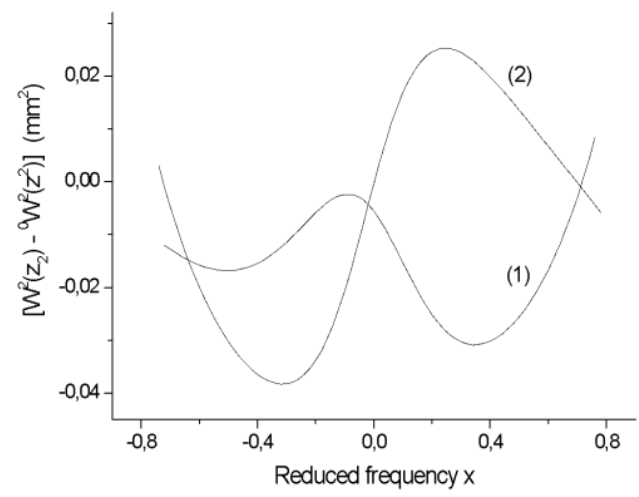

(b)

Figure 3: (a) Line shape and (b) variations of the quantity $\left[\mathrm{W}^{2}\left(\mathrm{z}_{2}\right)-{ }^{0} \mathrm{~W}^{2}\left(\mathrm{z}^{2}\right)\right]$ versus the reduced frequency for two positions of tube: (1) near the plane mirror, (2) near the concave mirror.

Fig. $3 \mathrm{a}$ and $3 \mathrm{~b}$ show respectively the line shapes obtained for two positions of the tube (plane side and curved side), and the variations of the corresponding mode diameter. As it can be seen, the line shape, initially of HF type becomes LF type when the tube is brought from the plane mirror to the concave mirror. The Garside effect plays, in this case, its entire role as shown in Fig.3b. The variation of asymmetry obtained implies the existence of an optimal position for which the line shape becomes 
symmetrical. In the following we will characterize the rate of the asymmetry by $\eta$ which is calculated according to $\eta=2\left(I_{H F}-I_{L F}\right) /\left(I_{H F}+I_{L F}\right)$, where $I_{H F}$ and $I_{L F}$ are the maximums of power at the high and low frequencies, respectively. The variations of $\eta$ as a function of the position $z_{3}$ of the tube allows the determination of the position $z_{3 . o p t}$ which gives $\eta=0$ corresponding to a symmetrical line shape. This gives $z_{3 . o p t}=174 \mathrm{~mm}$. However, it must be verified that, for this optimal position, the line shape remains symmetrical, even when the value $G$ of the gain is changed. Fig.4a illustrates this situation. Unlike the previous model, the line shape keeps its symmetry, even for high gains. This model shows clearly that we don't have a competition between the population and saturation effects, but rather a total cancellation of the variations of the mode diameter. This is confirmed through Fig. $4 \mathrm{~b}$ where the line shapes are perfectly symmetrical with respect to the center frequency. The Garside effect therefore acts in the same way for the low and high frequencies and the line shape keeps its symmetry. We can now say that, for this particular choice of cavity geometry, the effects of population and saturation inhomogeneities which are at the origin of the line shape asymmetry, do not compensate each other as it was the case in the model presented in reference [12] but are canceled simultaneously. The cavity is thus stabilized with respect to its transverse parameter and, in this case, the frequency of the Lamb-dip center matches exactly with the laser resonant frequency. This may be useful for the use of such a cavity as a standard of length and frequency and can be used in spectroscopy of materials as a reference for frequency measurements.

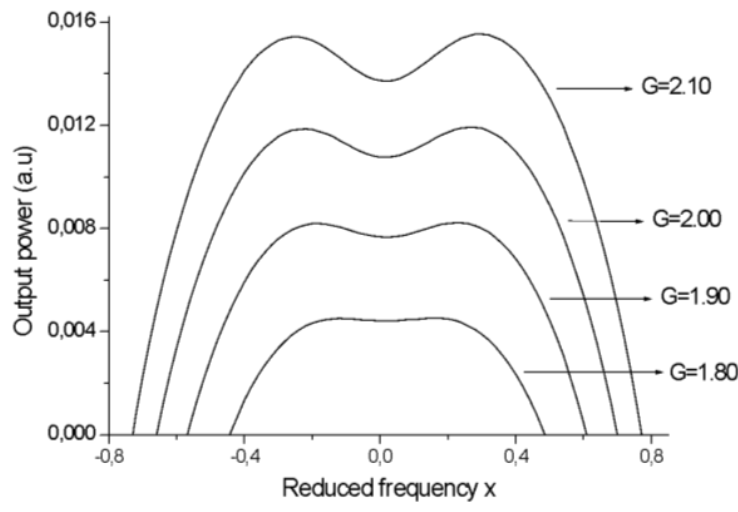

(a)

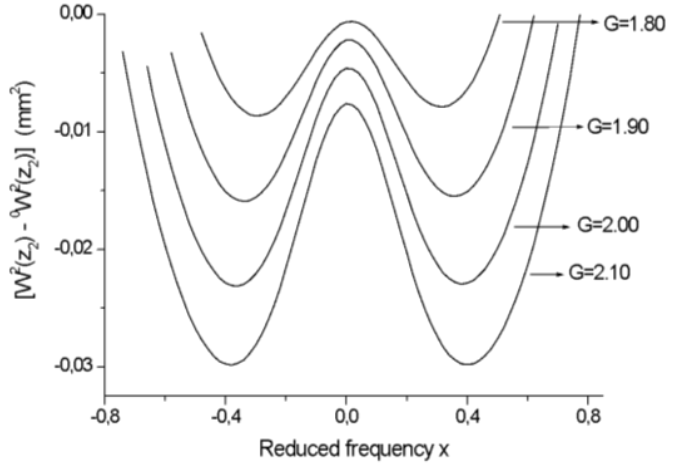

(b)

Figure 4: (a) Line shape and (b) variations of the quantity $\left[\mathrm{W}^{2}\left(\mathrm{z}_{2}\right)-{ }^{0} \mathrm{~W}^{2}\left(\mathrm{z}_{2}\right)\right]$ versus the reduced frequency for several gain values.

\section{Conclusion}

This study has highlighted the influence of several interdependent parameters on the line asymmetry. This complexity limits the use of simple theoretical models obtained from analytical processing. The saturation and population inhomogeneities are at the origin of the asymmetry of the line shape. However, the geometrical parameters of the cavity and the gain influence these two types of inhomogeneities and compete to favor one or the other asymmetry type. In some favorable cases, the two effects compensate each other, for a given gain, and the line shape is symmetrical. This corresponds to a simple compensation of antagonistic effects Unfortunately, the symmetry disappears as soon as the gain varies

Through the model we developed in the present work, we have shown, that a judicious choice of the geometrical parameters of the cavity, allows to cancel simultaneously the effects due to the population and the saturation inhomogeneities. The approach adopted allowed us to obtain a geometric configuration giving rise to a symmetrical line shape at the output of the cavity, and whatever the variation of the value of the gain. The obtained cavity is therefore well and truly stabilized. 


\section{References}

1. A. Aissani, B. Meziane, K. Battou and O. Ziane, "Spectral-line asymmetry in double-outputcavity lasers", Optics \& Laser Technology, vol. 42, no. 2, pp. 439-446, Oct 2009.

2. Z.D. Sun, R.M Lees and L.H. Xu, "Precision Lamb-dip infrared spectra of the $C$ - $N$ stretching band of $\mathrm{CH}_{3} \mathrm{NH}_{2}$ with a $\mathrm{CO}_{2}$-laser microwave-sideband spectrometer". J. Chem. Phys., vol. 132, 1-6, May 2010.

3. Z.D Sun, and al., "Lamb-dip spectroscopy of the $C-N$ stretching band of methylamine by using frequency-tunable microwave sidebands of $\mathrm{CO}_{2}$ laser lines", Sci. Rep.,vol. 6, 34270; doi: 10.1038/srep34270, 2016.

4. S. Welzel, F. Hempel, M. Hübner,N. Lang, P. B. Davies and J. Röpcke, "Quantum Cascade Laser Absorption Spectroscopy as a Plasma Diagnostic Tool: An Overview”,Sensors, vol 10, no.7, pp.6861-6900, 2010.

5. S. L. Ma, S. Picard, M. Zucco, J.M. Chartier, L. Robertsson, R.S. Windeler," Direct measurement of the absolute frequency of the international reference laser BIPM4" Metrologia, vol. 41, pp. 658, 2004.

6. A. Szoke, A. Javan, "Effects of collisions on saturation behaviour of the 1.15 um transition of Ne studied with He-Ue laser" Phys Rev, vol.145, no.1, pp. 137-147, May1966.

7. R.H. Cordover, P.A. Bouczyk, "Effects of Collisions on the Saturation Behavior of the $6328 A^{\circ}$ Transition of Ne Studied with a He-Ne Laser", Phys Rev, vol. 188, no.2, pp. 696-700, Dec 1969.

8. A.Szoke, A. Javan, "Isotope Shift and Saturation Behavior of the 1.15- $\mu$ Transition of Ne”, Phys. Rev. Lett. vol. 10, no. 12, pp. 521-524, Jun. 1963.

9. A. V. Miranov, “Observation of a Symmetrical Lamb Dip for a He-Ne Laser with a Natural Mixture of Neon Isotopes" Optics and Spectroscopy, vol. 92, pp. 473-474, no 3, March 2002.

10. G.Stephan, H. Taleb, F. Legros, C. Pesty, “Mise en évidence expérimentale et théorique de deux causes de dissymétrie dans la raie d'émission d'un laser à gaz” J. Phys. vol. 43, pp. 255 - 261, 1982.

11. G. Stephan, H. Trumper "Gaussian beam parameters and their stabilization in gas lasers”, Phys. Rev. A, vol. 28, no.6, pp.3450-3458, Dec 1983.

12. A. Le Floch, J.M. Lenormand, R. LE Naour andJ. P. Tache, » a critical geometry for lasers with internal lenslike Effects" J. Phys. Lett.vol. 43, no 14, pp. 493-498, 1982.

13. P. Cerez, R. Felder, " Gas-lens effect and cavity design of some frequency-stabilized He-Ne lasers”, Appl. Opt. vol. 22, pp. 1251-1256, 1983.

14. G. Stephan, H. Trumper, "Inhomogeneity Effects in a Gas Laser”, Phys. Rev. vol. A28, no.4, pp.2344-2362, Oct. 1983.

15. A. Kellou, G. Stephan, "Etude du champ proche d'un laser diaphragmé”, Applied Optics Vol. 26 (1987) 76-90. 